\title{
Frataxin and endothelial cell senescence in pulmonary hypertension
}

\author{
Allan Lawrie and Sheila E. Francis \\ Department of Infection, Immunity and Cardiovascular Disease, University of Sheffield, Sheffield, United Kingdom.
}

\begin{abstract}
Pulmonary hypertension (PH), increased blood pressure within the lungs, is classified into five diagnostic groups based on etiology, with treatment assigned on this basis. Currently, only Group 1 pulmonary arterial hypertension (PAH) and Group 4 chronic thromboembolic PH (CTEPH) have pharmacological treatments available. The role of the endothelial cell in pulmonary hypertension has long been debated, and in this issue of the JCI, Culley et al. present evidence for the reduction in frataxin expression across multiple groups of $\mathrm{PH}$. Reduced frataxin expression led to endothelial cell senescence and associated with the development of PH. Removal of the senescent cells using the senolytic drug Navitoclax in multiple models of PH effectively treated PH, suggesting a new class of treatments that may work beyond Group 1 and Group $4 \mathrm{PH}$ in patients with evidence of pulmonary vascular endothelial senescence.
\end{abstract}

\section{The physiological changes that result in pulmonary hypertension}

Pulmonary hypertension (PH) is defined by an increase in blood pressure within the pulmonary circulation. The cause of this increase in pulmonary artery blood pressure defines what type of $\mathrm{PH}$ an individual is suffering from, and whether there are any treatment options. $\mathrm{PH}$ is divided into five groups based on etiology, defined most recently at the 6th World Symposium on Pulmonary Hypertension in 2018 as Group 1 , pulmonary arterial hypertension $(\mathrm{PAH})$; Group 2, PH due to left heart disease; Group 3 , PH due to lung diseases and/or hypoxia; Group 4, PH due to pulmonary artery obstruction; and Group 5, PH with unclear and/or multifactorial mechanisms (1).

Paradoxically, when we think about $\mathrm{PH}$, we tend to imagine a young female presenting with breathlessness, dizziness, peripheral edema, and chest pain during activity who reports a high pulmonary artery pressure at right heart catheterization, with no obvious cause-so-called idiopathic pulmonary arterial hypertension (IPAH). In reality, this stereotype, although devastating and life-limiting for the affected individual, by far reflects the minority of cases across the whole spectrum of $\mathrm{PH}$. Indeed, while IPAH and the even broader classification of PAH are classified as rare diseases, $\mathrm{PH}$ as a complication of underlying cardiovascular or respiratory disease is extremely common. However, our quest to understand the pathophysiological and molecular changes that result in $\mathrm{PH}$ have largely focused on Group $1 \mathrm{PAH}$, and more precisely IPAH. It is rationalized that understanding the molecular pathogenesis of IPAH or PAH will allow us to extrapolate to other groups of $\mathrm{PH}$. In fact, we have made vast leaps in our understanding of the genetic and molecular sequelae leading to $\mathrm{PAH}$, but until relatively recently,

Delated Article: https://doi.org/10.1172/JCI136459

Conflict of interest: AL is funded by Alexion Pharmaceuticals, Janssen, GlaxoSmithKline, Novartis, IQVIA, and Apple, and receives personal fees from Janssen-Cilag and IP2IPO Australia Pty. AL, with The University of Sheffield, has been granted intellectual property entitled “Pulmonary Hypertension" (CB2510524/US9334327/JP2014532637)

Copyright: () 2021, American Society for Clinical Investigation.

Reference information: / Clin Invest. 2021;131(11):e149721. https://doi.org/10.1172/JCI149721.

therapies developed for PAH have yet to translate to the other $\mathrm{PH}$ groups. The first pharmacological treatment shown to have benefit outside of PAH was the soluble guanylate cyclase stimulator, riociguat, for Group 4 chronic thromboembolic pulmonary hypertension in the CHEST 1 study (2). Most recently, the INCREASE study demonstrated the benefit of inhaled treprostinil in patients with pulmonary hypertension due to interstitial lung disease (3). However, both of these treatments are pharmacological vasodilators that serve to reduce the afterload and strain on the right ventricle rather than addressing the central pathobiological problem of vascular remodeling. The molecular mechanisms regulating the pulmonary vascular remodeling process that drives $\mathrm{PAH}$ have been well described and include endothelial cell dysfunction, proliferation and migration of VSMCs and fibroblasts, and the infiltration of inflammatory cells. Importantly, pulmonary vascular remodeling also occurs in animal models of Group $3 \mathrm{PH}$, smoking-induced emphysema (4), and Group 2 $\mathrm{PH}$ and left heart failure-induced $\mathrm{PH}$ (5), offering hope that treatments targeting pulmonary vascular remodeling processes may offer benefit beyond Group 1 PAH.

\section{Dysfunctional endothelial cell signaling}

The role of the endothelial cell in pulmonary vascular remodeling was initially ignored. Researchers focused squarely on the vascular smooth muscle cells and adventitial fibroblasts, as these cells make up the vast majority of the medial, neointimal, and adventitial remodeling that drives the progressive narrowing luminal area or the pulmonary arterial tree. However, the observation of early endothelial cell apoptosis through to the emergence of apoptotic-resistant proliferative endothelial cells, endothelial cell senescence, and endothelial-to-mesenchymal cell transition within the pulmonary vasculature highlight the multifaceted role that endothelial cells can 


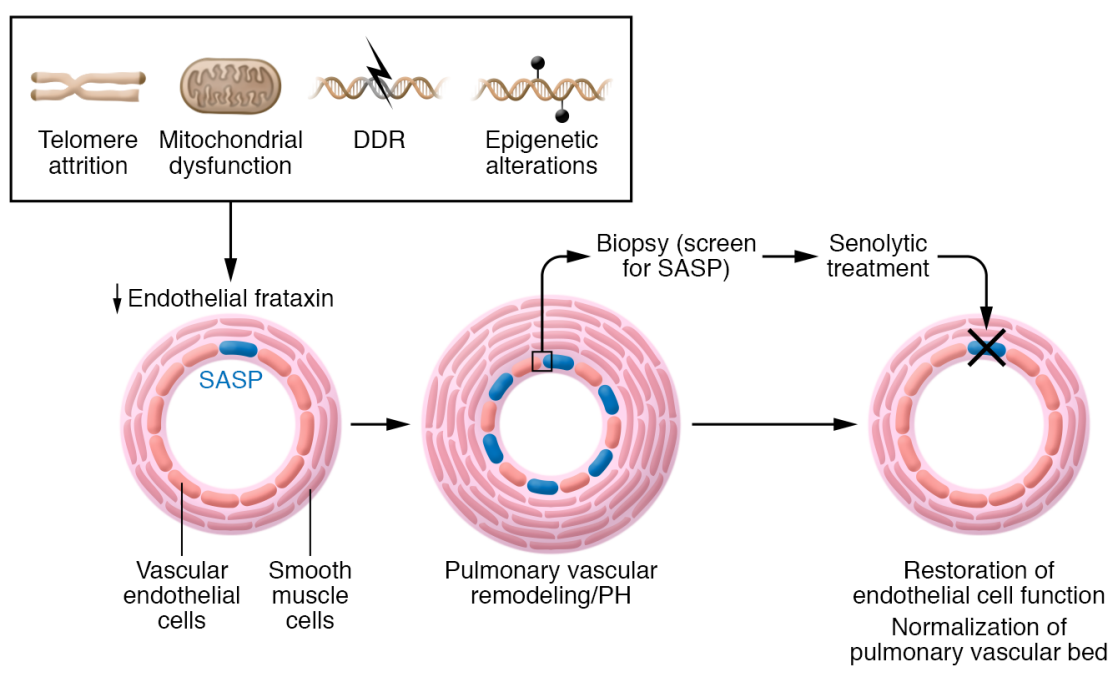

play in different stages of disease pathogenesis. A number of studies have highlighted the therapeutic potential of targeting dysfunctional endothelial cell signaling to reduce endothelial injury and reverse pulmonary vascular remodeling, including but not limited to inhibiting leukotriene B4 (6), osteoprotegerin (7), and addressing dysfunctional BMP/TGF- $\beta$ signaling (8-10).

In this issue of the JCI, Culley et al. (11) present further evidence that regulation of endothelial cell phenotype, specifically endothelial senescence, is a substantial driver of pulmonary vascular remodeling in multiple forms of $\mathrm{PH}$, straddling Groups 1-3. The manuscript builds on the authors' previous observations that a deficiency, specifically in endothelial cells, of iron-sufur (Fe-S) clusters can induce mitochondrial dysfunction and promote $\mathrm{PH}(12-14)$. Frataxin is a mitochondrial protein and iron chaperone essential for Fe-S clusters. Intriguingly, homozygous loss-of-function mutations in frataxin have been linked with Friedrich's ataxia, a neurodegenerative disease that can associate with hypertrophic cardiomyopathy (common cause of death). Since patients with hypertrophic cardiomyopathy can also develop Group 2 $\mathrm{PH}$, the authors hypothesized that endothelial frataxin deficiency would promote Fe-S-mediated stress/DNA damage-induced senescence and thereby lead to $\mathrm{PH}$. To test this hypothesis, the authors performed a comprehensive series of experiments utilizing patient material and animal models. A reduction in frataxin expression associated with an increase in $\mathrm{p} 16^{\mathrm{INK} 4}$, a marker of cell cycle arrest and senescence, in lung sections from patients with $\mathrm{PAH}$ and Group $3 \mathrm{PH}$, and animal models of $\mathrm{PAH}$ and Group $2 \mathrm{PH}$. Further, endothelial cells isolated from patients with PAH using single-cell RNASeq demonstrated distinct populations that expressed either markers of proliferation or senescence, with frataxin expression negatively correlating with $\mathrm{p} 16^{\mathrm{INK} 4}$ expression. siRNA-induced knock down of frataxin in endothelial cells confirmed this relationship. Additionally, induced pluripotent stem cells derived from patients with Friedreich's ataxia that were differentiated into endothelial cells (iPSC-ECs) exhibited cell cycle arrest and senescence. The authors subsequently demonstrate that endothelial frataxin deficiency (endothelial-specific frataxin knockout mice and siRNA) increased senescence and worsened $\mathrm{PH}$ in response to hypoxia. Finally, and importantly from a translational perspective, the authors then demonstrated that targeting endothelial senescence with senolytic therapy (Navitoclax) reversed established $\mathrm{PH}$ in multiple mouse models representing Group 1 and Group 3 PH. The series of experiments presented by Culley and colleagues (11) provide evidence for a role of frataxin-mediated EC senescence in multiple forms of $\mathrm{PH}$, and highlights a tantalizing treatment potential beyond Group $1 \mathrm{PAH}$.

\section{Clinical implications and precision medicine}

The importance of endothelial senescence is already appreciated in related vascular conditions, such as cardiovascular (15) and neurovascular disease (16) where
Figure 1. Model for precision medicine approaches to treating PH. Reduced endothelial cell frataxin levels caused by mutations or age-associated changes in telomere length, mitochondrial function, DNA damage, and the epigenome, can induce senescence. Accumulated endothelial cells exhibiting a prooxidant SASP release proinflammatory, profibrotic, and prosenescence factors that can remodel the pulmonary vascular and result in $\mathrm{PH}$. Clinicians may screen patient biopsies for senescent endothelial cells by staining with $\beta$-galactosidase and determining $p 16^{\text {INKA }}$ protein levels. Senolytic agents could potentially remove senescent cells, restore endothelial cell function, and normalize the pulmonary vascular bed.

senescent cells accumulate, exhibiting a prooxidant and senescence-associated phenotype (SASP) and releasing proinflammatory, profibrotic and prosenescence factors. Notably, senescent cells release the proinflammatory cytokine interleukin-1 (also implicated in PH, refs. 17-19), which can regulate endothelial lifespan $(20,21)$. Studies with senolytic agents in experimental models similar to the current study show the clinical potential of removing senescent cells in chronic conditions (Figure 1).

The ability for hypoxia and genetic and epigenetic regulation of frataxin to act as disease triggers fits well with the wider context of $\mathrm{PH}$ pathogenesis. Aside from the pathological expansion of a GAA triplet repeat within the first intron of both alleles of the frataxin gene $(F X N)$ that causes Friedreich's ataxia, there is no evidence to date of any genetic variants being associated with $\mathrm{PH}$. However, large genetic studies have so far concentrated on heritable or idiopathic PAH (22) or Group 1 PAH (23) when looking for causal or associated genetic variants. Whether there are variants that more subtly regulate frataxin perhaps warrants further investigation.

In Friedreich's ataxia, Libri et al. (24) demonstrated that patients treated daily with nicotinamide (vitamin B3), a class III HDAC inhibitor, upregulated sustained frataxin protein. Given the role of HDAC and interest in HDAC inhibitors in PAH (25), physician scientists may also consider nicotinamide treatment for $\mathrm{PH}$.

Looking to the future, and thinking about precision medicine approaches 
where treatment is administered not based on a clinical classification group but rather on a molecular phenotype, we can speculate that biomarkers of aging/senescence, e.g. telomere length in endothelial cells taken from the pulmonary artery at the time of diagnostic right heart catheterization, could individualize treatment choices to include pharmacological clearance of senescent cells, induced by frataxin deficiency or by other mechanisms (Figure 1).

\section{Acknowledgments}

AL is funded by a British Heart Foundation (BHF) Senior Basic Science Research Fellowship FS/18/52/33808, and he receives research support from BHF and Medical Research Council UK (UKRI). SEF received research funding from the $\mathrm{BHF}$ (PG 13/44/30365 and PG/20/10010).

Address correspondence to: Allan Lawrie, IICD, Medical School, Beech Hill Road, Sheffield S10 2RX, United Kingdom. Phone:44.0.114.215.9536;Email:a.lawrie@ sheffield.ac.uk.

1. Simonneau G, et al. Haemodynamic definitions and updated clinical classification of pulmonary hypertension. Eur Respir J. 2019;53(1):1801913.

2. Ghofrani H-A, et al. Riociguat for the treatment of chronic thromboembolic pulmonary hypertension. N Engl J Med. 2013;369(4):319-329.

3. Waxman A, et al. Inhaled treprostinil in pulmonary hypertension due to interstitial lung disease. N Engl J Med.2021;384(4):325-334.
4. Seimetz M, et al. Inducible NOS inhibition reverses tobacco-smoke-induced emphysema and pulmonary hypertension in mice. Cell. 2011;147(2):293-305.

5. Chen Y, et al. Left ventricular failure produces profound lung remodeling and pulmonary hypertension in mice: heart failure causes severe lung disease. Hypertension. 2012;59(6):1170-1178.

6. Tian W, et al. Blocking macrophage leukotriene b4 prevents endothelial injury and reverses pulmonary hypertension. Sci Transl Med. 2013;5(200):200ra117.

7. Arnold ND, et al. A therapeutic antibody targeting osteoprotegerin attenuates severe experimental pulmonary arterial hypertension. Nat Commun. 2019;10(1):5183.

8. Spiekerkoetter E, et al. FK506 activates BMPR2, rescues endothelial dysfunction, and reverses pulmonary hypertension. J Clin Invest. 2013;123(8):3600-3613.

9. Long L, et al. Selective enhancement of endothelial BMPR-II with BMP9 reverses pulmonary arterial hypertension. Nat Med. 2015;21(7):777-785.

10. Ryanto GRT, et al. An endothelial activin A-bone morphogenetic protein receptor type 2 link is overdriven in pulmonary hypertension. Nat Commun. 2021;12(1):1720.

11. Culley MK, et al. Frataxin deficiency promotes endothelial senescence in pulmonary hypertension. J Clin Invest. 2021;131(11):e136459.

12. Chan SY, et al. MicroRNA-210 controls mitochondrial metabolism during hypoxia by repressing the iron-sulfur cluster assembly proteins ISCU1/2. Cell Metab. 2009;10(4):273-284.

13. White $\mathrm{K}$, et al. Genetic and hypoxic alterations of the microRNA-210-ISCU1/2 axis promote iron-sulfur deficiency and pulmonary hypertension. EMBO Mol Med. 2015;7(6):695-713.

14. Yu Q, et al. BOLA (BolA Family Member 3) deficiency controls endothelial metabolism and glycine homeostasis in pulmonary hypertension. Circulation. 2019;139(19):2238-2255.
15. Childs BG, et al. Senescent cells: a therapeutic target for cardiovascular disease. J Clin Invest. 2018;128(4):1217-1228.

16. Baker DJ, Petersen RC. Cellular senescence in brain aging and neurodegenerative diseases: evidence and perspectives. J Clin Invest. 2018;128(4):1208-1216.

17. Humbert $M$, et al. Increased interleukin-1 and interleukin-6 serum concentrations in severe primary pulmonary hypertension. Am J Respir Crit Care Med. 1995;151(5):1628-1631.

18. Lawrie A, et al. Paigen diet-fed apolipoprotein E knockout mice develop severe pulmonary hypertension in an interleukin-1-dependent manner. Am J Pathol. 2011;179(4):1693-1705.

19. Pickworth J, et al. Differential IL-1 signaling induced by BMPR2 deficiency drives pulmonary vascular remodeling. Pulm Circ. 2017;7(4):768-776.

20. Maier JA, et al. Extension of the life-span of human endothelial cells by an interleukin-1 alpha antisense oligomer. Science. 1990;249(4976):1570-1574.

21. Dewberry RM, et al. Interleukin-1 receptor antagonist (IL-1RN) genotype modulates the replicative capacity of human endothelial cells. Circ Res. 2003;92(12):1285-1287.

22. Gräf S, et al. Identification of rare sequence variation underlying heritable pulmonary arterial hypertension. Nat Commun. 2018;9(1):1416.

23. Rhodes CJ, et al. Genetic determinants of risk in pulmonary arterial hypertension: international genome-wide association studies and meta-analysis. Lancet Respir Med. 2019;7(3):227-238.

24. Libri V, et al. Epigenetic and neurological effects and safety of high-dose nicotinamide in patients with Friedreich's ataxia: an exploratory, open-label, dose-escalation study. Lancet. 2014;384(9942):504-513.

25. Zhao L, et al. Histone deacetylation inhibition in pulmonary hypertension: therapeutic potential of valproic acid and suberoylanilide hydroxamic acid. Circulation. 2012;126(4):455-467. 